\title{
Rough $I_{2}$-lacunary statistical convergence of double sequences
}

\author{
Ömer Kişi ${ }^{1 *}$ and Erdinç Dündar ${ }^{2}$
}

\author{
"Correspondence: \\ okisi@bartin.edu.tr \\ ${ }^{1}$ Department of Mathematics, \\ Faculty of Science, Bartın University, \\ Bartın, Turkey \\ Full list of author information is \\ available at the end of the article
}

\begin{abstract}
In this paper, we introduce and study the notion of rough $\mathcal{I}_{2}$-lacunary statistical convergence of double sequences in normed linear spaces. We also introduce the notion of rough $\mathcal{I}_{2}$-lacunary statistical limit set of a double sequence and discuss some properties of this set.
\end{abstract}

Keywords: Statistical convergence; $\mathcal{I}$-convergence; Rough convergence; Lacunary sequences; Double sequences

\section{Introduction}

Throughout the paper, $\mathbb{N}$ and $\mathbb{R}$ denote the set of all positive integers and the set of all real numbers, respectively. The concept of convergence of a sequence of real numbers has been extended to statistical convergence independently by Fast [1] and Schoenberg [2]. This concept was extended to the double sequences by Mursaleen and Edely [3]. Lacunary statistical convergence was defined by Fridy and Orhan [4]. Çakan and Altay [5] presented multidimensional analogues of the results presented by Fridy and Orhan [4].

The idea of $\mathcal{I}$-convergence was introduced by Kostyrko et al. [6] as a generalization of statistical convergence which is based on the structure of the ideal $\mathcal{I}$ of subset of the set of natural numbers. Kostyrko et al. [7] studied the idea of $\mathcal{I}$-convergence and extremal $\mathcal{I}$-limit points. Das et al. [8,9] introduced the concept of $\mathcal{I}$-convergence of double sequences in a metric space and studied some properties of this convergence. A lot of development have been made in area about statistical convergence, $\mathcal{I}$-convergence and double sequences after the work of [1,2, 10-28].

The notion of lacunary ideal convergence of real sequences was introduced in [29]. Das et al. $[30,31]$ introduced new notions, namely $\mathcal{I}$-statistical convergence and $\mathcal{I}$-lacunary statistical convergence by using ideal. Belen et al. [32] introduced the notion of ideal statistical convergence of double sequences, which is a new generalization of the notions of statistical convergence and usual convergence. Kumar et al. [33] introduced I-lacunary statistical convergence of double sequences. Further investigation and applications on this notion can be found in [34].

The idea of rough convergence was first introduced by Phu [35] in finite-dimensional normed spaces. In another paper [36] related to this subject, Phu defined the rough continuity of linear operators and showed that every linear operator $f: X \rightarrow Y$ is $r$-continuous at every point $x \in X$ under the assumption $\operatorname{dim} Y<\infty$ and $r>0$, where $X$ and $Y$ are normed

(c) The Author(s) 2018. This article is distributed under the terms of the Creative Commons Attribution 4.0 International License (http://creativecommons.org/licenses/by/4.0/), which permits unrestricted use, distribution, and reproduction in any medium, provided you give appropriate credit to the original author(s) and the source, provide a link to the Creative Commons license, and indicate if changes were made. 
spaces. In [37], Phu extended the results given in [35] to infinite-dimensional normed spaces. Aytar [38] studied the rough statistical convergence. Also, Aytar [39] studied that the rough limit set and the core of a real sequence. Recently, Dündar and Çakan [11, 40], Pal et al. [41] introduced the notion of rough $\mathcal{I}$-convergence and the set of rough $\mathcal{I}$-limit points of a sequence and studied the notion of rough convergence and the set of rough limit points of a double sequence. Further this notion of rough convergence of double sequence has been extended to rough statistical convergence of double sequence by Malik et al. [42] using double natural density of $\mathbb{N} \times \mathbb{N}$ in the similar way as the notion of convergence of double sequence in Pringsheim sense was generalized to statistical convergence of double sequence. Also, Dündar [43] investigated rough $\mathcal{I}_{2}$-convergence of double sequences. The notion of $\mathcal{I}$-statistical convergence of double sequences was introduced by Malik and Ghosh [44] in the theory of rough convergence.

In view of the recent applications of ideals in the theory of convergence of sequences, it seems very natural to extend the interesting concept of rough lacunary statistical convergence further by using ideals which we mainly do here.

So it is quite natural to think, if the new notion of $\mathcal{I}$-lacunary statistical convergence of double sequences can be introduced in the theory of rough convergence.

\section{Definitions and notations}

In this section, we recall some definitions and notations, which form the base for the present study $[6,10,11,23,32,33,35,38,40,42-46]$.

Throughout the paper, let $r$ be a nonnegative real number and $\mathbb{R}^{n}$ denotes the real $n$ dimensional space with the norm $\|\cdot\|$. Consider a sequence $x=\left(x_{i}\right) \subset \mathbb{R}^{n}$.

The sequence $x=\left(x_{i}\right)$ is said to be $r$-convergent to $x_{*}$, denoted by $x_{i} \stackrel{r}{\longrightarrow} x_{*}$, provided that

$$
\forall \varepsilon>0 \exists i_{\varepsilon} \in \mathbb{N}: i \geq i_{\varepsilon} \quad \Rightarrow \quad\left\|x_{i}-x_{*}\right\|<r+\varepsilon
$$

The set

$$
\operatorname{LIM}^{r} x:=\left\{x_{*} \in \mathbb{R}^{n}: x_{i} \stackrel{r}{\longrightarrow} x_{*}\right\}
$$

is called the $r$-limit set of the sequence $x=\left(x_{i}\right)$. A sequence $x=\left(x_{i}\right)$ is said to be $r$ convergent if $\operatorname{LIM}^{r} x \neq \emptyset$. In this case, $r$ is called the convergence degree of the sequence $x=\left(x_{i}\right)$. For $r=0$, we get the ordinary convergence. There are several reasons for this interest (see [35]).

A family of sets $\mathcal{I} \subseteq 2^{\mathbb{N}}$ is called an ideal if and only if

(i) $\emptyset \in \mathcal{I}$,

(ii) for each $A, B \in \mathcal{I}$ we have $A \cup B \in \mathcal{I}$,

(iii) for each $A \in \mathcal{I}$ and each $B \subseteq A$ we have $B \in \mathcal{I}$.

An ideal is called non-trivial if $\mathbb{N} \notin \mathcal{I}$ and a non-trivial ideal is called admissible if $\{n\} \in \mathcal{I}$ for each $n \in \mathbb{N}$.

A family of sets $\mathcal{F} \subseteq 2^{\mathbb{N}}$ is a filter in $\mathbb{N}$ if and only if

(i) $\emptyset \notin \mathcal{F}$,

(ii) for each $A, B \in \mathcal{F}$ we have $A \cap B \in \mathcal{F}$,

(iii) for each $A \in \mathcal{F}$ and each $B \supseteq A$ we have $B \in \mathcal{F}$. 
If $\mathcal{I}$ is a non-trivial ideal in $\mathbb{N}$ ( i.e., $\mathbb{N} \notin \mathcal{I}$ ), then the family of sets

$$
\mathcal{F}(\mathcal{I})=\{M \subset \mathbb{N}: \exists A \in \mathcal{I}: M=\mathbb{N} \backslash A\}
$$

is a filter of $\mathbb{N}$ and it is called the filter associated with the ideal $\mathcal{I}$.

A sequence $x=\left(x_{i}\right)$ is said to be rough $\mathcal{I}$-convergent $\left(r\right.$ - $\mathcal{I}$-convergent) to $x_{*}$ with the roughness degree $r$, denoted by $x_{i} \stackrel{r-\mathcal{I}}{\longrightarrow} x_{*}$ provided that $\left\{i \in \mathbb{N}:\left\|x_{i}-x_{*}\right\| \geq r+\varepsilon\right\} \in \mathcal{I}$ for every $\varepsilon>0$; or equivalently, if the condition

$$
\mathcal{I} \text { - } \lim \sup \left\|x_{i}-x_{*}\right\| \leq r
$$

is satisfied. In addition, we can write $x_{i} \stackrel{r-\mathcal{I}}{\longrightarrow} x_{*}$ iff the inequality $\left\|x_{i}-x_{*}\right\|<r+\varepsilon$ holds for every $\varepsilon>0$ and almost all $i$.

A double sequence $x=\left(x_{m n}\right)_{(m, n) \in \mathbb{N} \times \mathbb{N}}$ of real numbers is said to be bounded if there exists a positive real number $M$ such that $\left|x_{m n}\right|<M$, for all $m, n \in \mathbb{N}$. That is

$$
\|x\|_{\infty}=\sup _{m, n}\left|x_{m n}\right|<\infty
$$

A double sequence $x=\left(x_{m n}\right)$ of real numbers is said to be convergent to $L \in \mathbb{R}$ in Pringsheim's sense (shortly, $p$-convergent to $L \in \mathbb{R}$ ), if for any $\varepsilon>0$, there exists $N_{\varepsilon} \in \mathbb{N}$ such that $\left|x_{m n}-L\right|<\varepsilon$, whenever $m, n>N_{\varepsilon}$. In this case, we write

$$
\lim _{m, n \rightarrow \infty} x_{m n}=L
$$

We recall that a subset $K$ of $\mathbb{N} \times \mathbb{N}$ is said to have natural density $d(K)$ if

$$
d(K)=\lim _{m, n \rightarrow \infty} \frac{K(m, n)}{m \cdot n},
$$

where $K(m, n)=|\{(j, k) \in \mathbb{N} \times \mathbb{N}: j \leq m, k \leq n\}|$.

Throughout the paper we consider a sequence $x=\left(x_{m n}\right)$ such that $\left(x_{m n}\right) \in \mathbb{R}^{n}$.

Let $x=\left(x_{m n}\right)$ be a double sequence in a normed space $(X,\|\cdot\|)$ and $r$ be a non-negative real number. $x$ is said to be $r$-statistically convergent to $\xi$, denoted by $x \stackrel{r \text {-st }}{\longrightarrow} \xi$, if for $\varepsilon>0$ we have $d(A(\varepsilon))=0$, where $A(\varepsilon)=\left\{(m, n) \in \mathbb{N} \times \mathbb{N}:\left\|x_{m n}-\xi\right\| \geq r+\varepsilon\right\}$. In this case, $\xi$ is called the $r$-statistical limit of $x$.

A non-trivial ideal $\mathcal{I}_{2}$ of $\mathbb{N} \times \mathbb{N}$ is called strongly admissible if $\{i\} \times \mathbb{N}$ and $\mathbb{N} \times\{i\}$ belong to $\mathcal{I}_{2}$ for each $i \in \mathbb{N}$.

It is evident that a strongly admissible ideal is admissible also.

Throughout the paper we take $\mathcal{I}_{2}$ as a strongly admissible ideal in $\mathbb{N} \times \mathbb{N}$.

Let $(X, \rho)$ be a metric space A double sequence $x=\left(x_{m n}\right)$ in $X$ is said to be $\mathcal{I}_{2}$-convergent to $L \in X$, if for any $\varepsilon>0$ we have $A(\varepsilon)=\left\{(m, n) \in \mathbb{N} \times \mathbb{N}: \rho\left(x_{m n}, L\right) \geq \varepsilon\right\} \in \mathcal{I}_{2}$. In this case, we say that $x$ is $\mathcal{I}_{2}$-convergent and we write

$$
\mathcal{I}_{2^{-}} \lim _{m, n \rightarrow \infty} x_{m n}=L
$$


A double sequence $x=\left(x_{m n}\right)$ is said to be rough convergent ( $r$-convergent) to $x_{*}$ with the roughness degree $r$, denoted by $x_{m n} \stackrel{r}{\longrightarrow} x_{*}$ provided that

$$
\forall \varepsilon>0 \exists k_{\varepsilon} \in \mathbb{N}: m, n \geq k_{\varepsilon} \quad \Rightarrow \quad\left\|x_{m n}-x_{*}\right\|<r+\varepsilon,
$$

or equivalently, if

$$
\lim \sup \left\|x_{m n}-x_{*}\right\| \leq r
$$

A double sequence $x=\left(x_{m n}\right)$ is said to be $r$ - $\mathcal{I}_{2}$-convergent to $x_{*}$ with the roughness degree $r$, denoted by $x_{m n} \stackrel{r-\mathcal{I}_{2}}{\longrightarrow} x_{*}$ provided that

$$
\left\{(m, n) \in \mathbb{N} \times \mathbb{N}:\left\|x_{m n}-x_{*}\right\| \geq r+\varepsilon\right\} \in \mathcal{I}_{2},
$$

for every $\varepsilon>0$; or equivalently, if the condition

$$
\mathcal{I}_{2}-\lim \sup \left\|x_{m n}-x_{*}\right\| \leq r
$$

is satisfied. In addition, we can write $x_{m n} \stackrel{r-\mathcal{I}_{2}}{\longrightarrow} x_{*}$ iff the inequality $\left\|x_{m n}-x_{*}\right\|<r+\varepsilon$ holds for every $\varepsilon>0$ and almost all $(m, n)$.

Now, we give the definition of $\mathcal{I}_{2}$-asymptotic density of $\mathbb{N} \times \mathbb{N}$.

A subset $K \subset \mathbb{N} \times \mathbb{N}$ is said to be have $\mathcal{I}_{2}$-asymptotic density $d_{\mathcal{I}_{2}}(K)$ if

$$
d_{\mathcal{I}_{2}}(K)=\mathcal{I}_{2}-\lim _{m, n \rightarrow \infty} \frac{|K(m, n)|}{m \cdot n}
$$

where $K(m, n)=\{(j, k) \in \mathbb{N} \times \mathbb{N}: j \leq m, k \leq n ;(j, k) \in K\}$ and $|K(m, n)|$ denotes number of elements of the set $K(m, n)$.

A double sequence $x=\left\{x_{j k}\right\}$ of real numbers is $\mathcal{I}_{2}$-statistically convergent to $\varepsilon$, and we write $x \stackrel{\mathcal{I}_{2} \text {-st }}{\rightarrow} \xi$, provided that for any $\varepsilon>0$ and $\delta>0$

$$
\left\{(m, n) \in \mathbb{N} \times \mathbb{N}: \frac{1}{m n}\left|\left\{(j, k):\left\|x_{j k}-\xi\right\| \geq \varepsilon, j \leq m, k \leq n\right\}\right| \geq \delta\right\} \in \mathcal{I}_{2} .
$$

Let $x=\left\{x_{j k}\right\}$ be a double sequence in a normed linear space $(X,\|\cdot\|)$ and $r$ be a non-negative real number. Then $x$ is said to be rough $\mathcal{I}_{2}$-statistical convergent to $\xi$ or $r$ - $\mathcal{I}_{2}$-statistical convergent to $\xi$ if for any $\varepsilon>0$ and $\delta>0$

$$
\left\{(m, n) \in \mathbb{N} \times \mathbb{N}: \frac{1}{m n}\left|\left\{(j, k), j \leq m, k \leq n:\left\|x_{j k}-\xi\right\| \geq r+\varepsilon\right\}\right| \geq \delta\right\} \in \mathcal{I}_{2} .
$$

In this case, $\xi$ is called the rough $\mathcal{I}_{2}$-statistical limit of $x=\left\{x_{j k}\right\}$ and we denote it by $x \stackrel{r-\mathcal{I}_{2} \text {-st }}{\longrightarrow} \xi$.

A double sequence $\bar{\theta}=\theta_{u s}=\left\{\left(k_{u}, l_{s}\right)\right\}$ is called a double lacunary sequence if there exist two increasing sequences of integers $\left(k_{u}\right)$ and $\left(l_{s}\right)$ such that

$$
k_{0}=0, \quad h_{u}=k_{u}-k_{u-1} \rightarrow \infty \quad \text { and } \quad l_{0}=0, \quad \bar{h}_{s}=l_{s}-l_{s-1} \rightarrow \infty, \quad u, s \rightarrow \infty .
$$


We will use the notation $k_{u s}:=k_{u} l_{s}, h_{u s}:=h_{u} \bar{h}_{s}$ and $\theta_{u s}$ is determined by

$$
\begin{aligned}
& J_{u s}:=\left\{(k, l): k_{u-1}<k \leq k_{u} \text { and } l_{s-1}<l \leq l_{s}\right\}, \\
& q_{u}:=\frac{k_{u}}{k_{u-1}}, \quad \bar{q}_{s}:=\frac{l_{s}}{l_{s-1}} \quad \text { and } \quad q_{u s}:=q_{u} \bar{q}_{s} .
\end{aligned}
$$

Throughout the paper, by $\theta_{2}=\theta_{u s}=\left\{\left(k_{u}, l_{s}\right)\right\}$ we will denote a double lacunary sequence of positive real numbers, respectively, unless otherwise stated.

A double sequence $x=\left\{x_{m n}\right\}$ of numbers is said to be $\mathcal{I}_{2}$-lacunary statistical convergent or $S_{\theta_{2}}\left(\mathcal{I}_{2}\right)$-convergent to $L$, if for each $\varepsilon>0$ and $\delta>0$,

$$
\left\{(u, s) \in \mathbb{N} \times \mathbb{N}: \frac{1}{h_{u s}}\left|\left\{(m, n) \in J_{u s}:\left|x_{m n}-L\right| \geq \varepsilon\right\}\right| \geq \delta\right\} \in \mathcal{I}_{2} .
$$

In this case, we write $x_{m n} \rightarrow L\left(S_{\theta_{2}}\left(\mathcal{I}_{2}\right)\right)$ or $S_{\theta_{2}}\left(\mathcal{I}_{2}\right)$ - $\lim _{m, n \rightarrow \infty} x_{m n}=L$.

\section{Main results}

Definition 3.1 Let $x=\left\{x_{j k}\right\}$ be a double sequence in a normed linear space $(X,\|\cdot\|)$ and $r$ be a non-negative real number. Then $x$ is said to be rough lacunary statistical convergent to $\xi$ or $r$-lacunary statistical convergent to $\xi$ if for any $\varepsilon>0$

$$
\lim _{u, s \rightarrow \infty} \frac{1}{h_{u s}}\left|\left\{(j, k) \in J_{u s}:\left\|x_{j k}-\xi\right\| \geq r+\varepsilon\right\}\right|=0 .
$$

In this case $\xi$ is called the rough lacunary statistical limit of $x=\left\{x_{j k}\right\}$ and we denote it by $x \stackrel{r-S_{\theta_{2}}}{\longrightarrow} \xi$.

Definition 3.2 Let $x=\left\{x_{j k}\right\}$ be a double sequence in a normed linear space $(X,\|\cdot\|)$ and $r$ be a non-negative real number. Then $x$ is said to be rough $\mathcal{I}_{2}$-lacunary statistical convergent to $\xi$ or $r$ - $\mathcal{I}_{2}$-lacunary statistical convergent to $\xi$ if for any $\varepsilon>0$ and $\delta>0$

$$
\left\{(u, s) \in \mathbb{N} \times \mathbb{N}: \frac{1}{h_{u s}}\left|\left\{(j, k) \in J_{u s}:\left\|x_{j k}-\xi\right\| \geq r+\varepsilon\right\}\right| \geq \delta\right\} \in \mathcal{I}_{2}
$$

In this case, $\xi$ is called the rough $\mathcal{I}_{2}$-lacunary statistical limit of $x=\left\{x_{j k}\right\}$ and we denote it by $x \stackrel{r-\mathcal{I}_{\theta_{2}} \text {-st }}{\longrightarrow} \xi$.

Remark 3.3 Note that if $\mathcal{I}_{2}$ is the ideal

$$
\mathcal{I}_{2}^{0}=\{A \subset \mathbb{N} \times \mathbb{N}: \exists m(A) \in \mathbb{N} \text { such that } i, j \geq m(A) \Rightarrow(i, j) \notin A\},
$$

then rough $\mathcal{I}_{2}$-lacunary statistical convergence coincides with rough lacunary statistical convergence.

Here $r$ in the above definition is called the roughness degree of the rough $\mathcal{I}_{2}$-lacunary statistical convergence. If $r=0$, we obtain the notion of $\mathcal{I}_{2}$-lacunary convergence. But our main interest is when $r>0$. It may happen that a double sequence $x=\left\{x_{j k}\right\}$ is not $\mathcal{I}_{2}$ lacunary statistical convergent in the usual sense, but there exists a double sequence $y=$ 
$\left\{y_{j k}\right\}$, which is $\mathcal{I}_{2}$-lacunary statistically convergent and satisfying the condition $\left\|x_{j k}-y_{j k}\right\| \leq$ $r$ for all $(j, k)$. Then $x$ is rough $\mathcal{I}_{2}$-lacunary statistically convergent to the same limit.

From the above definition it is clear that the rough $\mathcal{I}_{2}$-lacunary statistical limit of a double sequence is not unique. So we consider the set of rough $\mathcal{I}_{2}$-lacunary statistical limits of a double sequence $x$ and we use the notation $\mathcal{I}_{\theta_{2}}$-st- $\operatorname{LIM}_{x}^{r}$ to denote the set of all rough $\mathcal{I}_{2}$-lacunary statistical limits of a double sequence $x$. We say that a double sequence $x$ is rough $\mathcal{I}_{2}$-lacunary statistically convergent if $\mathcal{I}_{\theta_{2}}$-st- $\operatorname{LIM}_{x}^{r} \neq \emptyset$.

Throughout the paper $X$ denotes a normed linear space $(X,\|\cdot\|)$ and $x$ denotes the double sequence $\left\{x_{j k}\right\}$ in $X$.

Now, we discuss some basic properties of rough $\mathcal{I}_{2}$-lacunary statistically convergence of double sequences.

Theorem 3.4 Let $x=\left\{x_{j k}\right\}$ be a double sequence and $r \geq 0$. Then $\mathcal{I}_{\theta_{2}}$-st- $\operatorname{LIM}_{x}^{r} \leq 2 r$. In particular if $x$ is rough $\mathcal{I}_{2}$-lacunary statistically convergent to $\xi$, then

$$
\mathcal{I}_{\theta_{2}}-s t-\operatorname{LIM}_{x}^{r}=\overline{B_{r}(\xi)}
$$

where $\overline{B_{r}(\xi)}=\{y \in X:\|y-\xi\| \leq r\}$ and so

$$
\operatorname{diam}\left(\mathcal{I}_{\theta_{2}} \text {-st- } \mathrm{LIM}_{x}^{r}\right)=2 r
$$

Proof Let $\operatorname{diam}\left(\mathcal{I}_{\theta_{2}}\right.$-st- $\left.\operatorname{LIM}_{x}^{r}\right)>2 r$. Then there exist $y, z \in \mathcal{I}_{\theta_{2}}$-st-LIM ${ }_{x}^{r}$ such that $\|y-z\|>$ $2 r$. Now, we select $\varepsilon>0$ so that $\varepsilon<\frac{\|y-z\|}{2}-r$. Let

$$
A=\left\{(j, k) \in J_{u s}:\left\|x_{j k}-y\right\| \geq r+\varepsilon\right\}
$$

and

$$
B=\left\{(j, k) \in J_{u s}:\left\|x_{j k}-z\right\| \geq r+\varepsilon\right\} .
$$

Then

$$
\begin{aligned}
& \frac{1}{h_{u s}}\left|\left\{(j, k) \in J_{u s}:(j, k) \in A \cup B\right\}\right| \\
& \quad \leq \frac{1}{h_{u s}}\left|\left\{(j, k) \in J_{u s}:(j, k) \in A\right\}\right|+\frac{1}{h_{u s}}\left|\left\{(j, k) \in J_{u s}:(j, k) \in B\right\}\right|,
\end{aligned}
$$

and so by the property of $\mathcal{I}_{2}$-convergence

$$
\begin{aligned}
& \mathcal{I}_{2-} \lim _{u, s \rightarrow \infty} \frac{1}{h_{u s}}\left|\left\{(j, k) \in J_{u s}:(j, k) \in A \cup B\right\}\right| \\
& \leq \mathcal{I}_{2-} \lim _{u, s \rightarrow \infty} \frac{1}{h_{u s}}\left|\left\{(j, k) \in J_{u s}:(j, k) \in A\right\}\right| \\
& \quad+\mathcal{I}_{2-} \lim _{u, s \rightarrow \infty} \frac{1}{h_{u s}}\left|\left\{(j, k) \in J_{u s}:(j, k) \in B\right\}\right| \\
& =0 .
\end{aligned}
$$


Thus,

$$
\left\{(u, s) \in \mathbb{N} \times \mathbb{N}: \frac{1}{h_{u s}}\left|\left\{(j, k) \in J_{u s}:(j, k) \in A \cup B\right\}\right| \geq \delta\right\} \in \mathcal{I}_{2}
$$

for all $\delta>0$. Let

$$
H=\left\{(u, s) \in \mathbb{N} \times \mathbb{N}: \frac{1}{h_{u s}}\left|\left\{(j, k) \in J_{u s}:(j, k) \in A \cup B\right\}\right| \geq \frac{1}{2}\right\} .
$$

Clearly $H \in \mathcal{I}_{2}$, so choose $\left(u_{0}, s_{0}\right) \in \mathbb{N} \times \mathbb{N} \backslash H$. Then

$$
\frac{1}{h_{u_{0} s_{0}}}\left|\left\{(j, k) \in J_{u s}:(j, k) \in A \cup B\right\}\right|<\frac{1}{2} \text {. }
$$

So, we have

$$
\frac{1}{h_{u_{0} s_{0}}}\left|\left\{(j, k) \in J_{u s}:(j, k) \notin A \cup B\right\}\right| \geq 1-\frac{1}{2}=\frac{1}{2},
$$

i.e., $\left\{(j, k) \in J_{u s}:(j, k) \notin A \cup B\right\}$ is a nonempty set.

Take $\left(j_{0}, k_{0}\right) \in J_{u s}$ such that $\left(j_{0}, k_{0}\right) \notin A \cup B$. Then $\left(j_{0}, k_{0}\right) \in A^{c} \cap B^{c}$ and so $\left\|x_{j_{0} k_{0}}-y\right\|<r+\varepsilon$ and $\left\|x_{j_{0} k_{0}}-z\right\|<r+\varepsilon$. Hence, we have

$$
\begin{aligned}
\|y-z\| & \leq\left\|x_{j_{0} k_{0}}-y\right\|+\left\|x_{j_{0} k_{0}}-z\right\| \\
& \leq 2(r+\varepsilon) \\
& \leq\|y-z\|,
\end{aligned}
$$

which is absurd. Therefore, $\mathcal{I}_{\theta_{2}}$-st- $\operatorname{LIM}_{x}^{r} \leq 2 r$.

If $\mathcal{I}_{\theta_{2}}$-st- $\operatorname{LIM}_{x}^{r}=\xi$, then we proceed as follows. Let $\varepsilon>0$ and $\delta>0$ be given. Then

$$
A=\left\{(u, s) \in \mathbb{N} \times \mathbb{N}: \frac{1}{h_{u s}}\left|\left\{(j, k) \in J_{u s}:\left\|x_{j k}-\xi\right\| \geq \varepsilon\right\}\right| \geq \delta\right\} \in \mathcal{I}_{2} .
$$

Then for $(u, s) \notin A$ we have

$$
\frac{1}{h_{u s}}\left|\left\{(j, k) \in J_{u s}:\left\|x_{j k}-\xi\right\| \geq \varepsilon\right\}\right|<\delta,
$$

i.e.,

$$
\frac{1}{h_{u s}}\left|\left\{(j, k) \in J_{u s}:\left\|x_{j k}-\xi\right\|<\varepsilon\right\}\right| \geq 1-\delta .
$$

Now, for each $y \in \overline{B_{r}(\xi)}$ we have

$$
\left\|x_{j k}-y\right\| \leq\left\|x_{j k}-\xi\right\|+\|\xi-y\| \leq\left\|x_{j k}-\xi\right\|+r .
$$

Let

$$
B_{u s}=\left\{(j, k) \in J_{u s}:\left\|x_{j k}-\xi\right\|<\varepsilon\right\} .
$$


Then for $(j, k) \in B_{u s}$ we have $\left\|x_{j k}-y\right\|<r+\varepsilon$. Hence, we have

$$
B_{u s}=\left\{(j, k) \in J_{u s}:\left\|x_{j k}-y\right\|<r+\varepsilon\right\} .
$$

This implies

$$
\frac{\left|B_{u s}\right|}{h_{u s}} \leq \frac{1}{h_{u s}}\left|\left\{(j, k) \in J_{u s}:\left\|x_{j k}-y\right\|<r+\varepsilon\right\}\right|,
$$

i.e.,

$$
\frac{1}{h_{u s}}\left|\left\{(j, k) \in J_{u s}:\left\|x_{j k}-y\right\|<r+\varepsilon\right\}\right| \geq 1-\delta .
$$

Thus, for all $(j, k) \notin A$,

$$
\frac{1}{h_{u s}}\left|\left\{(j, k) \in J_{u s}:\left\|x_{j k}-y\right\| \geq r+\varepsilon\right\}\right|<1-(1-\delta)
$$

and so we have

$$
\left\{(u, s) \in \mathbb{N} \times \mathbb{N}: \frac{1}{h_{u s}}\left|\left\{(j, k) \in J_{u s} ;\left\|x_{j k}-y\right\| \geq r+\varepsilon\right\}\right| \geq \delta\right\} \subset A .
$$

Since $A \in \mathcal{I}_{2}$

$$
\left\{(u, s) \in \mathbb{N} \times \mathbb{N}: \frac{1}{h_{u s}}\left|\left\{(j, k) \in J_{u s}:\left\|x_{j k}-y\right\| \geq r+\varepsilon\right\}\right| \geq \delta\right\} \in \mathcal{I}_{2} .
$$

This shows that $y \in \mathcal{I}_{\theta_{2}}$-st- $\operatorname{LIM}_{x}^{r}$. Therefore, $\mathcal{I}_{\theta_{2}}$-st- $\operatorname{LIM}_{x}^{r} \supset \overline{B_{r}(\xi)}$.

Conversely, let $y \in \mathcal{I}_{\theta_{2}}$-st- $\operatorname{LIM}_{x}^{r},\|y-\xi\|>r$ and $\varepsilon=\frac{\|y-\xi\|-r}{2}$. Now, we take

$$
M_{1}=\left\{(j, k) \in J_{u s}:\left\|x_{j k}-y\right\| \geq r+\varepsilon\right\}
$$

and

$$
M_{2}=\left\{(j, k) \in J_{u s}:\left\|x_{j k}-\xi\right\| \geq \varepsilon\right\} .
$$

Then

$$
\begin{aligned}
& \frac{1}{h_{u s}}\left|\left\{(j, k) \in J_{u s}:(j, k) \in M_{1} \cup M_{2}\right\}\right| \\
& \quad \leq \frac{1}{h_{u s}}\left|\left\{(j, k) \in J_{u s}:(j, k) \in M_{1}\right\}\right|+\frac{1}{h_{u s}}\left|\left\{(j, k) \in J_{u s}:(j, k) \in M_{2}\right\}\right|,
\end{aligned}
$$

and by the property of $\mathcal{I}_{2}$-convergence

$$
\begin{gathered}
\mathcal{I}_{2}-\lim _{u, s \rightarrow \infty} \frac{1}{h_{u s}}\left|\left\{(j, k) \in J_{u s}:(j, k) \in M_{1} \cup M_{2}\right\}\right| \\
\quad=\mathcal{I}_{2^{-}} \lim _{u, s \rightarrow \infty} \frac{1}{h_{u s}}\left|\left\{(j, k) \in J_{u s}:(j, k) \in M_{1}\right\}\right|
\end{gathered}
$$




$$
\begin{aligned}
& \quad+\mathcal{I}_{2-} \lim _{u, s \rightarrow \infty} \frac{1}{h_{u s}}\left|\left\{(j, k) \in J_{u s}:(j, k) \in M_{2}\right\}\right| \\
& =0 .
\end{aligned}
$$

Now, we let

$$
M=\left\{(u, s) \in \mathbb{N} \times \mathbb{N}: \frac{1}{h_{u s}}\left|\left\{(j, k):(j, k) \in M_{1} \cup M_{2}\right\}\right| \geq \frac{1}{2}\right\} .
$$

Clearly $M \in \mathcal{I}_{2}$ and we choose $\left(u_{0}, s_{0}\right) \in \mathbb{N} \times \mathbb{N} \backslash M$. Then we have

$$
\frac{1}{h_{u_{0} s_{0}}}\left|\left\{(j, k):(j, k) \in M_{1} \cup M_{2}\right\}\right|<\frac{1}{2}
$$

and so

$$
\frac{1}{h_{u_{0} s_{0}}}\left|\left\{(j, k):(j, k) \notin M_{1} \cup M_{2}\right\}\right| \geq 1-\frac{1}{2}=\frac{1}{2},
$$

i.e., $\left\{(j, k):(j, k) \notin M_{1} \cup M_{2}\right\}$ is a nonempty set. Let $\left(j_{0}, k_{0}\right) \in J_{u s}$ such that $\left(j_{0}, k_{0}\right) \notin M_{1} \cup M_{2}$. Then $\left(j_{0}, k_{0}\right) \in M_{1}^{c} \cap M_{2}^{c}$ and hence $\left\|x_{j_{0} k_{0}}-y\right\|<r+\varepsilon$ and $\left\|x_{j_{0} k_{0}}-\xi\right\|<\varepsilon$. So

$$
\|y-\xi\| \leq\left\|x_{j_{0} k_{0}}-y\right\|+\left\|x_{j_{0} k_{0}}-\xi\right\| \leq r+2 \varepsilon \leq\|y-\xi\|,
$$

which is absurd. Therefore, $\|y-\xi\| \leq r$ and so $y \in \overline{B_{r}(\xi)}$. Consequently, we have

$$
\mathcal{I}_{\theta_{2}} \text {-st- } \operatorname{LIM}_{x}^{r}=\overline{B_{r}(\xi)}
$$

Theorem 3.5 Let $x=\left\{x_{j k}\right\}$ be a double sequence and $r \geq 0$ be a real number. Then the rough $\mathcal{I}_{2}$-lacunary statistical limit set of the double sequence $x$, i.e., the set $\mathcal{I}_{\theta_{2}}$-st- $\mathrm{LIM}_{x}^{r}$ is closed.

Proof If $\mathcal{I}_{\theta_{2}}$-st- $\operatorname{LIM}_{x}^{r}=\emptyset$, then there is nothing to prove.

Let us assume that $\mathcal{I}_{\theta_{2}}$-st- $\operatorname{LIM}_{x}^{r} \neq \emptyset$. Now, consider a double sequence $\left\{y_{j k}\right\}$ in $\mathcal{I}_{\theta_{2}}$-st- $\operatorname{LIM}_{x}^{r}$ with $\lim _{j, k \rightarrow \infty} y_{j k}=y$. Choose $\varepsilon>0$ and $\delta>0$. Then there exists $i_{\frac{\varepsilon}{2}} \in \mathbb{N}$ such that for all $j, k \geq i \frac{\varepsilon}{2}$

$$
\left\|y_{j k}-y\right\|<\frac{\varepsilon}{2}
$$

Let $j_{0}, k_{0}>i_{\frac{\varepsilon}{2}}$. Then $y_{j_{0} k_{0}} \in \mathcal{I}_{\theta_{2}}$-st- $\operatorname{LIM}_{x}^{r}$. Consequently, we have

$$
A=\left\{(u, s) \in \mathbb{N} \times \mathbb{N}: \frac{1}{h_{u s}}\left|\left\{(j, k) \in J_{u s} ;\left\|x_{j k}-y_{j_{0} k_{0}}\right\| \geq r+\frac{\varepsilon}{2}\right\}\right| \geq \delta\right\} \in \mathcal{I}_{2}
$$

Clearly $M=\mathbb{N} \times \mathbb{N} \backslash A$ is nonempty, choose $(u, s) \in M$. We have

$$
\frac{1}{h_{u s}}\left|\left\{(j, k) \in J_{u s}:\left\|x_{j k}-y_{j_{0} k_{0}}\right\| \geq r+\frac{\varepsilon}{2}\right\}\right|<\delta
$$


and so

$$
\frac{1}{h_{u s}}\left|\left\{(j, k) \in J_{u s}:\left\|x_{j k}-y_{j k_{0}}\right\|<r+\frac{\varepsilon}{2}\right\}\right| \geq 1-\delta .
$$

Put

$$
B_{u s}=\left\{(j, k) \in J_{u s}:\left\|x_{j k}-y_{j_{0} k_{0}}\right\|<r+\frac{\varepsilon}{2}\right\}
$$

and select $(j, k) \in B_{u s}$. Then we have

$$
\begin{aligned}
\left\|x_{j k}-y\right\| & \leq\left\|x_{j k}-y_{j_{0} k_{0}}\right\|+\left\|y_{j_{0} k_{0}}-y\right\| \\
& <r+\frac{\varepsilon}{2}+\frac{\varepsilon}{2} \\
& =r+\varepsilon
\end{aligned}
$$

and so

$$
B_{u s} \subset\left\{(j, k) \in J_{u s}:\left\|x_{j k}-y\right\|<r+\varepsilon\right\},
$$

which implies

$$
1-\delta \leq \frac{\left|B_{u s}\right|}{h_{u s}} \leq \frac{1}{h_{u s}}\left|\left\{(j, k) \in J_{u s}:\left\|x_{j k}-y\right\|<r+\varepsilon\right\}\right| .
$$

Therefore,

$$
\frac{1}{h_{u s}}\left|\left\{(j, k) \in J_{u s}:\left\|x_{j k}-y\right\| \geq r+\varepsilon\right\}\right|<1-(1-\delta)=\delta
$$

and so we have

$$
\left\{(u, s) \in \mathbb{N} \times \mathbb{N}: \frac{1}{h_{u s}}\left|\left\{(j, k) \in J_{u s}:\left\|x_{j k}-y\right\| \geq r+\varepsilon\right\}\right| \geq \delta\right\} \subset A \in \mathcal{I}_{2} .
$$

This shows that $y \in \mathcal{I}_{\theta_{2}}$-st- $\operatorname{LIM}_{x}^{r}$. Hence, $\mathcal{I}_{\theta_{2}}$-st- $\operatorname{LIM}_{x}^{r}$ is a closed set.

Theorem 3.6 Let $x=\left\{x_{j k}\right\}$ be a double sequence and $r \geq 0$ be a real number. Then the rough $\mathcal{I}_{2}$-lacunary statistical limit set $\mathcal{I}_{\theta_{2}}$-st-LIM ${ }_{x}^{r}$ of the double sequence $x$ is a convex set.

Proof Let $y_{0}, y_{1} \in \mathcal{I}_{\theta_{2}}$-st- $\operatorname{LIM}_{x}^{r}$ and $\varepsilon>0$ be given. Let

$$
A_{0}=\left\{(j, k) \in J_{u s}:\left\|x_{j k}-y_{0}\right\| \geq r+\varepsilon\right\}
$$

and

$$
A_{1}=\left\{(j, k) \in J_{u s}:\left\|x_{j k}-y_{1}\right\| \geq r+\varepsilon\right\} .
$$


Then by Theorem 3.4, for $\delta>0$ we have

$$
\left\{(u, s) \in \mathbb{N} \times \mathbb{N}: \frac{1}{h_{u s}}\left|\left\{(j, k) \in J_{u s}:(j, k) \in A_{0} \cup A_{1}\right\}\right| \geq \delta\right\} \in \mathcal{I}_{2} .
$$

Now, we choose $0<\delta_{1}<1$ such that $0<1-\delta_{1}<\delta$ and let

$$
A=\left\{(u, s) \in \mathbb{N} \times \mathbb{N}: \frac{1}{h_{u s}}\left|\left\{(j, k) \in J_{u s}:(j, k) \in A_{0} \cup A_{1}\right\}\right| \geq 1-\delta_{1}\right\} .
$$

Then $A \in \mathcal{I}_{2}$. For all $(u, s) \notin A$, we have

$$
\frac{1}{h_{u s}}\left|\left\{(j, k) \in J_{u s}:(j, k) \in A_{0} \cup A_{1}\right\}\right|<1-\delta_{1}
$$

and so

$$
\frac{1}{h_{u s}}\left|\left\{(j, k) \in J_{u s}:(j, k) \notin A_{0} \cup A_{1}\right\}\right| \geq\left\{1-\left(1-\delta_{1}\right)\right\}=\delta_{1} .
$$

Therefore, $\left\{(j, k):(j, k) \notin A_{0} \cup A_{1}\right\}$ is a nonempty set. Let us take $\left(j_{0}, k_{0}\right) \in A_{0}^{c} \cap A_{1}^{c}$ and $0 \leq \mu \leq 1$. Then

$$
\begin{aligned}
\left\|x_{j_{0} k_{0}}-\left[(1-\mu) y_{0}+\mu y_{1}\right]\right\| & =\left\|(1-\mu) x_{j_{0} k_{0}}+\mu x_{j_{0} k_{0}}-\left[(1-\mu) y_{0}+\mu y_{1}\right]\right\| \\
& \leq(1-\mu)\left\|x_{j_{0} k_{0}}-y_{0}\right\|+\mu\left\|x_{j_{0} k_{0}}-y_{1}\right\| \\
& <(1-\mu)(r+\varepsilon)+\mu(r+\varepsilon)=r+\varepsilon .
\end{aligned}
$$

Let

$$
M=\left\{(j, k) \in J_{u s}:\left\|x_{j k}-\left[(1-\mu) y_{0}+\mu y_{1}\right]\right\| \geq r+\varepsilon\right\} .
$$

Then clearly, $A_{0}^{c} \cap A_{1}^{c} \subset M^{c}$. So for $(u, s) \notin A$, we have

$$
\delta_{1} \leq \frac{1}{h_{u s}}\left|\left\{(j, k) \in J_{u s}:(j, k) \notin A_{0} \cup A_{1}\right\}\right| \leq \frac{1}{h_{u s}}\left|\left\{(j, k) \in J_{u s}:(j, k) \notin M\right\}\right|
$$

and so

$$
\frac{1}{h_{u s}}\left|\left\{(j, k) \in J_{u s}:(j, k) \in M\right\}\right|<1-\delta_{1}<\delta .
$$

Therefore,

$$
A^{c} \subset\left\{(u, s) \in \mathbb{N} \times \mathbb{N}: \frac{1}{h_{u s}}\left|\left\{(j, k) \in J_{u s}:(j, k) \in M\right\}\right|<\delta\right\} .
$$

Since $A^{c} \in \mathcal{F}\left(\mathcal{I}_{2}\right)$, we have

$$
\left\{(u, s) \in \mathbb{N} \times \mathbb{N}: \frac{1}{h_{u s}}\left|\left\{(j, k) \in J_{u s}:(j, k) \in M\right\}\right|<\delta\right\} \in \mathcal{F}\left(\mathcal{I}_{2}\right)
$$


and so

$$
\left\{(u, s) \in \mathbb{N} \times \mathbb{N}: \frac{1}{h_{u s}}|\{(j, k):(j, k) \in M\}| \geq \delta\right\} \in \mathcal{I}_{2} .
$$

This completes the proof.

Theorem 3.7 A double sequence $x=\left\{x_{j k}\right\}$ is rough $\mathcal{I}_{2}$-lacunary statistical convergent to $\xi$ if and only if there exists a double sequence $y=\left\{y_{j k}\right\}$ such that $\mathcal{I}_{\theta_{2}}-$ st- $y=\xi$ and $\left\|x_{j k}-y_{j k}\right\| \leq r$, for all $(j, k) \in \mathbb{N} \times \mathbb{N}$.

Proof Let $y=\left\{y_{j k}\right\}$ be a double sequence in $X$, which is $\mathcal{I}_{2}$-lacunary statistically convergent to $\xi$ and $\left\|x_{j k}-y_{j k}\right\| \leq r$, for all $(j, k) \in \mathbb{N} \times \mathbb{N}$. Then for any $\varepsilon>0$ and $\delta>0$

$$
A=\left\{(u, s) \in \mathbb{N} \times \mathbb{N}: \frac{1}{h_{u s}}\left|\left\{(j, k) \in J_{u s}:\left\|y_{j k}-\xi\right\| \geq \varepsilon\right\}\right| \geq \delta\right\} \in \mathcal{I}_{2} .
$$

Let $(u, s) \notin A$. Then we have

$$
\frac{1}{h_{u s}}\left|\left\{(j, k) \in J_{u s}:\left\|y_{j k}-\xi\right\| \geq \varepsilon\right\}\right|<\delta \Rightarrow \frac{1}{h_{u s}}\left|\left\{(j, k) \in J_{u s}:\left\|y_{j k}-\xi\right\|<\varepsilon\right\}\right| \geq 1-\delta .
$$

Now, we let

$$
B_{u s}=\left\{(j, k) \in J_{u s}:\left\|y_{j k}-\xi\right\|<\varepsilon\right\} .
$$

Then, for $(j, k) \in B_{u s}$, we have

$$
\left\|x_{j k}-\xi\right\| \leq\left\|x_{j k}-y_{j k}\right\|+\left\|y_{j k}-\xi\right\|<r+\varepsilon,
$$

and so

$$
\begin{aligned}
B_{u s} & \subset\left\{(j, k) \in J_{u s}:\left\|x_{j k}-\xi\right\|<r+\varepsilon\right\} \\
& \Rightarrow \quad \frac{\left|B_{u s}\right|}{h_{u s}} \leq \frac{1}{h_{u s}}\left|\left\{(j, k) \in J_{u s}:\left\|x_{j k}-\xi\right\|<r+\varepsilon\right\}\right| \\
& \Rightarrow \frac{1}{h_{u s}}\left|\left\{(j, k) \in J_{u s}:\left\|x_{j k}-\xi\right\|<r+\varepsilon\right\}\right| \geq 1-\delta \\
& \Rightarrow \frac{1}{h_{u s}}\left|\left\{(j, k) \in J_{u s}:\left\|x_{j k}-\xi\right\| \geq r+\varepsilon\right\}\right|<1-(1-\delta)=\delta .
\end{aligned}
$$

Thus, we have

$$
\left\{(u, s) \in \mathbb{N} \times \mathbb{N}: \frac{1}{h_{u s}}\left|\left\{(j, k) \in J_{u s}:\left\|x_{j k}-\xi\right\| \geq r+\varepsilon\right\}\right| \geq \delta\right\} \subset A
$$

and, since $A \in \mathcal{I}_{2}$,

$$
\left\{(u, s) \in \mathbb{N} \times \mathbb{N}: \frac{1}{h_{u s}}\left|\left\{(j, k) \in J_{u s}:\left\|x_{j k}-\xi\right\| \geq r+\varepsilon\right\}\right| \geq \delta\right\} \in \mathcal{I}_{2} .
$$

Hence, $\mathcal{I}_{\theta_{2}}$-st- $y=\xi$. 
Conversely, suppose that $\mathcal{I}_{\theta_{2}}$-st- $y=\xi$. Then, for $\varepsilon>0$ and $\delta>0$,

$$
A=\left\{(u, s) \in \mathbb{N} \times \mathbb{N}: \frac{1}{h_{u s}}\left|\left\{(j, k) \in J_{u s}:\left\|x_{j k}-\xi\right\| \geq r+\varepsilon\right\}\right| \geq \delta\right\} \in \mathcal{I}_{2} .
$$

Let $(u, s) \notin A$. Then we have

$$
\frac{1}{h_{u s}}\left|\left\{(j, k) \in J_{u s}:\left\|x_{j k}-\xi\right\| \geq r+\varepsilon\right\}\right|<\delta
$$

and so

$$
\frac{1}{h_{u s}}\left|\left\{(j, k) \in J_{u s}:\left\|x_{j k}-\xi\right\|<r+\varepsilon\right\}\right| \geq 1-\delta .
$$

Let

$$
B_{u s}=\left\{(j, k) \in J_{u s}:\left\|x_{j k}-\xi\right\|<r+\varepsilon\right\} .
$$

Now, we define a double sequence $y=\left\{y_{j k}\right\}$ as follows:

$$
y_{j k}= \begin{cases}\xi, & \text { if }\left\|x_{j k}-\xi\right\| \leq r \\ x_{j k}+r \frac{\xi-x_{j k}}{\left\|x_{j k}-\xi\right\|}, & \text { otherwise. }\end{cases}
$$

Then

$$
\begin{aligned}
\left\|y_{j k}-\xi\right\| & = \begin{cases}0, & \text { if }\left\|x_{j k}-\xi\right\| \leq r, \\
\left\|x_{j k}-\xi+r \frac{\xi-x_{j k}}{\left\|x_{j k}-\xi\right\|}\right\|, & \text { otherwise, }\end{cases} \\
& = \begin{cases}0, & \text { if }\left\|x_{j k}-\xi\right\| \leq r, \\
\left\|x_{j k}-\xi\right\|-r, & \text { otherwise. }\end{cases}
\end{aligned}
$$

Let $(j, k) \in B_{u s}$. Then we have

$$
\left\|y_{j k}-\xi\right\|=0, \quad \text { if }\left\|x_{j k}-\xi\right\| \leq r \quad \text { and } \quad\left\|y_{j k}-\xi\right\|<\varepsilon, \quad \text { if } r<\left\|x_{j k}-\xi\right\|<r+\varepsilon
$$

and so

$$
B_{u s} \subset\left\{(j, k) \in J_{u s}:\left\|y_{j k}-\xi\right\|<\varepsilon\right\} .
$$

This implies

$$
\frac{\left|B_{u s}\right|}{h_{u s}} \leq \frac{1}{h_{u s}}\left|\left\{(j, k) \in J_{u s}:\left\|y_{j k}-\xi\right\|<\varepsilon\right\}\right| .
$$

Hence, we have

$$
\begin{aligned}
& \frac{1}{h_{u s}}\left|\left\{(j, k) \in J_{u s}:\left\|y_{j k}-\xi\right\|<\varepsilon\right\}\right| \geq 1-\delta \\
& \quad \Rightarrow \quad \frac{1}{h_{u s}}\left|\left\{(j, k) \in J_{u s}:\left\|y_{j k}-\xi\right\| \geq \varepsilon\right\}\right|<1-(1-\delta)=\delta
\end{aligned}
$$


and so

$$
\left\{(u, s) \in \mathbb{N} \times \mathbb{N}: \frac{1}{h_{u s}}\left|\left\{(j, k) \in J_{u s}:\left\|x_{j k}-\xi\right\| \geq \varepsilon\right\}\right| \geq \delta\right\} \subset A .
$$

Since $A \in \mathcal{I}_{2}$ we have

$$
\left\{(u, s) \in \mathbb{N} \times \mathbb{N}: \frac{1}{h_{u s}}\left|\left\{(j, k) \in J_{u s}:\left\|x_{j k}-\xi\right\| \geq \varepsilon\right\}\right| \geq \delta\right\} \in \mathcal{I}_{2} .
$$

So, $\mathcal{I}_{\theta_{2}}$-st- $y=\xi$.

Definition 3.8 A double sequence $x=\left\{x_{j k}\right\}$ is said to be $\mathcal{I}_{\theta_{2}}$-statistically bounded if there exists a positive number $K$ such that for any $\delta>0$ the set

$$
A=\left\{(u, s) \in \mathbb{N} \times \mathbb{N}: \frac{1}{h_{u s}}\left|\left\{(j, k) \in J_{u s}:\left\|x_{j k}\right\| \geq K\right\}\right| \geq \delta\right\} \in \mathcal{I}_{2} .
$$

The next result provides a relationship between boundedness and rough $\mathcal{I}_{\theta_{2}}$-statistical convergence of double sequences.

Theorem 3.9 If a double sequence $x=\left\{x_{j k}\right\}$ is bounded then there exists $r \geq 0$ such that $\mathcal{I}_{\theta_{2}}$ st- $\operatorname{LIM}_{x}^{r} \neq \emptyset$.

Proof Let $x=\left\{x_{j k}\right\}$ be bounded double sequence. There exists a positive real number $K$ such that $\left\|x_{j k}\right\|<K$, for all $(j, k) \in J_{u s}$. Let $\varepsilon>0$ be given. Then

$$
\left\{(j, k) \in J_{u s}:\left\|x_{j k}-0\right\| \geq K+\varepsilon\right\}=\emptyset .
$$

Therefore, $0 \in \mathcal{I}_{\theta_{2}}$-st- $\operatorname{LIM}_{x}^{K}$ and so $\mathcal{I}_{\theta_{2}}$-st- $\operatorname{LIM}_{x}^{K} \neq \emptyset$.

Remark 3.10 The converse of the above theorem is not true. For example, let us consider the double sequence $x=\left\{x_{j k}\right\}$ in $\mathbb{R}$ defined by

$$
x_{j k=} \begin{cases}j k, & \text { if } j \text { and } k \text { are squares } \\ 5, & \text { otherwise. }\end{cases}
$$

Then $\mathcal{I}_{\theta_{2}}$-st- $\operatorname{LIM}_{x}^{0}=\{5\} \neq \emptyset$ but the double sequence $x$ is unbounded.

Definition 3.11 A point $\lambda \in X$ is said to be an $\mathcal{I}_{2}$-lacunary statistical cluster point of a double sequence $x=\left\{x_{j k}\right\}$ in $X$ if for any $\varepsilon>0$

$$
d_{\mathcal{I}_{2}}\left(\left\{(j, k) \in J_{u s}:\left\|x_{j k}-\lambda\right\|<\varepsilon\right\}\right) \neq 0,
$$

where

$$
d_{\mathcal{I}_{2}}(A)=\mathcal{I}_{2-} \lim _{u, s \rightarrow \infty} \frac{1}{h_{u s}}\left|\left\{(j, k) \in J_{u s}:(j, k) \in A\right\}\right|,
$$

if it exists. The set of $\mathcal{I}_{2}$-lacunary statistical cluster points of $x$ is denoted by $\Lambda_{x}^{S_{\theta_{2}}}\left(\mathcal{I}_{2}\right)$. 
Theorem 3.12 For any arbitrary $\alpha \in \Lambda_{x}^{S_{\theta_{2}}}\left(\mathcal{I}_{2}\right)$ of a double sequence $x=\left\{x_{j k}\right\}$ we have $\|\xi-\alpha\| \leq r$, for all $\xi \in \mathcal{I}_{\theta_{2}}$-st- $\operatorname{LIM}_{x}^{r}$.

Proof Assume that there exists a point $\alpha \in \Lambda_{x}^{S_{\theta_{2}}}\left(\mathcal{I}_{2}\right)$ and $\xi \in \mathcal{I}_{\theta_{2}}$-st- $\operatorname{LIM}_{x}^{r}$ such that $\|\xi-\alpha\|>r$. Let $\varepsilon=\frac{\|\xi-\alpha\|-r}{3}$. Then

$$
\left\{(j, k) \in J_{u s}:\left\|x_{j k}-\xi\right\| \geq r+\varepsilon\right\} \supset\left\{(j, k) \in J_{u s}:\left\|x_{j k}-\alpha\right\|<\varepsilon\right\} .
$$

Since $\alpha \in \Lambda_{x}^{S_{\theta_{2}}}\left(\mathcal{I}_{2}\right)$ we have

$$
d_{\mathcal{I}_{2}}\left(\left\{(j, k) \in J_{u s}:\left\|x_{j k}-\alpha\right\|<\varepsilon\right\}\right) \neq 0 .
$$

Hence by (8) we have

$$
d_{\mathcal{I}_{2}}\left(\left\{(j, k) \in J_{u s}:\left\|x_{j k}-\alpha\right\| \geq r+\varepsilon\right\}\right) \neq 0,
$$

which contradicts that $\xi \in \mathcal{I}_{\theta_{2}}$-st- $\operatorname{LIM}_{x}^{r}$. Hence, $\|\xi-\alpha\| \leq r$.

\section{Conclusion}

The rough convergence has recently been studied by several authors. In view of the recent applications of ideals in the theory of convergence of sequences, it seems very natural to extend the interesting concept of rough lacunary statistical convergence further by using ideals, which we mainly do here; and we investigate some properties of this new type of convergence. So, we have extended some well-known results.

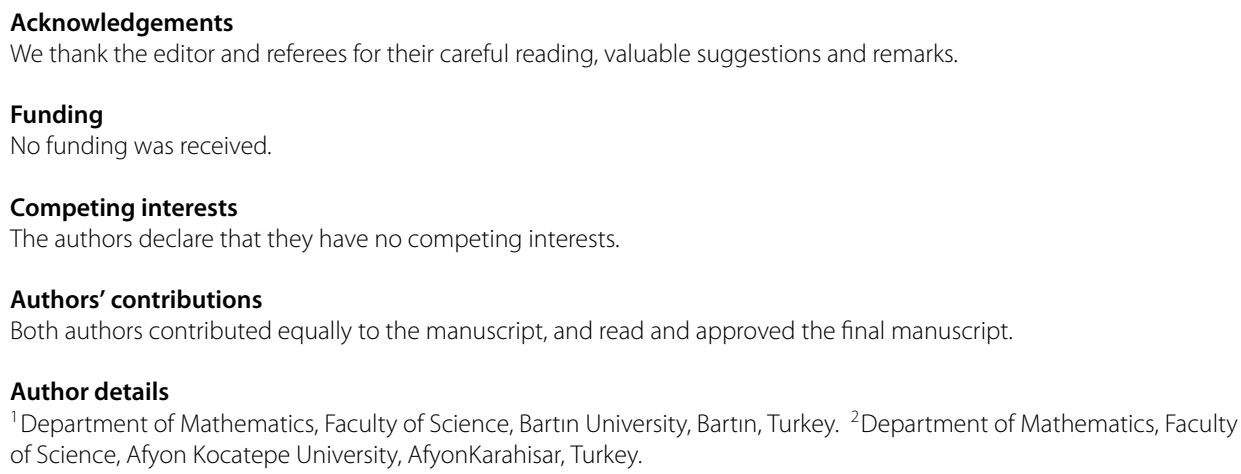

\section{Publisher's Note}

Springer Nature remains neutral with regard to jurisdictional claims in published maps and institutional affiliations.

Received: 18 June 2018 Accepted: 28 August 2018 Published online: 10 September 2018

\section{References}

1. Fast, H.: Sur la convergenc statistique. Colloq. Math. 2, 241-244 (1951)

2. Schoenberg, I.J.: The integrability of certain functions and related summability methods. Am. Math. Mon. 66, 361-375 (1959)

3. Mursaleen, M., Edely, O.H.H.: Statistical convergence of double sequences. J. Math. Anal. Appl. 288, $223-231$ (2003)

4. Fridy, J.A., Orhan, C.: Lacunary statistical convergence. Pac. J. Math. 160(1), 43-51 (1993)

5. Çakan, C., Altay, B.: Statistically boundedness and statistical core of double sequences. J. Math. Anal. Appl. 317(2), 690-697 (2006)

6. Kostyrko, P., Šalát, T., Wilczyński, W.: I-convergence. Real Anal. Exch. 26(2), 669-686 (2000)

7. Kostyrko, P., Macaj, M., Šalát, T., Sleziak, M.: I-convergence and extremal /-limit points. Math. Slovaca 55, 443-464 (2005) 
8. Das, P., Kostyrko, P., Wilczyński, W., Malik, P.: / and /*-convergence of double sequences. Math. Slovaca 58(5), 605-620 (2008)

9. Das, P., Malik, P.: On extremal / limit points of double sequences. Tatra Mt. Math. Publ. 40, 91-102 (2008)

10. Altay, B., Başar, F.: Some new spaces of double sequences. J. Math. Anal. Appl. 309(1), 70-90 (2005)

11. Dündar, E., Çakan, C.: Rough convergence of double sequences. Demonstr. Math. 47(3), 638-651 (2014)

12. Fridy, J.A.: On statistical convergence. Analysis 5, 301-313 (1985)

13. Gürdal, M., Huban, M.B.: On 1-convergence of double sequences in the topology induced by random 2-norms. Mat. Vesn. 66(1), 73-83 (2014)

14. Gürdal, M., Şahiner, A.: Extremal /-limit points of double sequences. Appl. Math. E-Notes 8, 131-137 (2008)

15. Miller, H.I.: A measure theoretical subsequence characterization of statistical convergence. Trans. Am. Math. Soc. 347(5), 1811-1819 (1995)

16. Mohiuddine, S.A., Alotaibi, A., Alsulami, S.M.: Ideal convergence of double sequences in random 2-normed spaces. Adv. Differ. Equ. 2012, Article ID 149 (2012)

17. Mohiuddine, S.A., Hazarika, B.: Some classes of ideal convergent sequences and generalized difference matrix operator. Filomat 31(6), 1827-1834 (2017)

18. Mursaleen, M., Mohiuddine, S.A., Edely, O.H.H.: On the ideal convergence of double sequences in intuitionistic fuzzy normed spaces. Comput. Math. Appl. 59,603-611 (2010)

19. Mursaleen, M., Mohiuddine, S.A.: On ideal convergence of double sequences in probabilistic normed spaces. Math Rep. 12(4), 359-371 (2010)

20. Mursaleen, M., Mohiuddine, S.A.: On ideal convergence in probabilistic normed spaces. Math. Slovaca 62(1), 49-62 (2012)

21. Mursaleen, M., Alotaibi, A.: On /-convergence in random 2-normed spaces. Math. Slovaca 61(6), $933-940$ (2011)

22. Nabiev, A., Pehlivan, S., Gürdal, M.: On I-Cauchy sequences. Taiwan. J. Math. 11(2), 569-576 (2007)

23. Pringsheim, A.: Zur theorie der zweifach unendlichen zahlenfolgen. Math. Ann. 53, 289-321 (1900)

24. Šalát, T., Tripathy, B.C., Ziman, M.: On I-convergence field. Ital. J. Pure Appl. Math. 17, 45-54 (2005)

25. Salát, T.: On statistically convergent sequences of real numbers. Math. Slovaca 30, 139-150 (1980)

26. Savaş, E., Gürdal, M.: I-statistical convergence in probabilistic normed spaces. Sci. Bull. "Politeh." Univ. Buchar., Ser. A, Appl. Math. Phys. 77(4), 195-204 (2015)

27. Demirci, K.: /-limit superior and limit inferior. Math. Commun. 6, 165-172 (2001)

28. Nuray, F., Ruckle, W.H.: Generalized statistical convergence and convergence free spaces. J. Math. Anal. Appl. 245, 513-527 (2000)

29. Tripathy, B.C., Hazarika, B., Choudhary, B.: Lacunary I-convergent sequences. Kyungpook Math. J. 52(4), 473-482 (2012)

30. Das, P., Savaş, E., Ghosal, S.K.: On generalizations of certain summability methods using ideals. Appl. Math. Lett. 24, 1509-1514 (2011)

31. Savaş, E., Das, P.A.: A generalized statistical convergence via ideals. Appl. Math. Lett. 24, 826-830 (2011)

32. Belen, C., Yıldırım, M.: On generalized statistical convergence of double sequences via ideals. Ann. Univ. Ferrara 58(1), $11-20(2012)$

33. Kumar, S., Kumar, V., Bhatia, S.S.: On ideal version of lacunary statistical convergence of double sequences. Gen. Math. Notes 17(1), 32-44 (2013)

34. Hazarika, B.: Lacunary ideal convergence of multiple sequences. J. Egypt. Math. Soc. 24(1), 54-59 (2016)

35. Phu, H.X.: Rough convergence in normed linear spaces. Numer. Funct. Anal. Optim. 22, 199-222 (2001)

36. Phu, H.X.: Rough continuity of linear operators. Numer. Funct. Anal. Optim. 23, 139-146 (2002)

37. Phu, H.X.: Rough convergence in infinite dimensional normed spaces. Numer. Funct. Anal. Optim. 24, $285-301$ (2003)

38. Aytar, S.: Rough statistical convergence. Numer. Funct. Anal. Optim. 29(3-4), 291-303 (2008)

39. Aytar, S.: The rough limit set and the core of a real sequence. Numer. Funct. Anal. Optim. 29(3-4), 283-290 (2008)

40. Dündar, E., Çakan, C.: Rough I-convergence. Gulf J. Math. 2(1), 45-51 (2014)

41. Pal, S.K., Chandra, D., Dutta, S.: Rough ideal convergence. Hacet. J. Math. Stat. 42(6), 513-527 (2000)

42. Malik, P., Maity, M.: On rough statistical convergence of double sequences in normed linear spaces. Afr. Math. 27, $141-148(2015)$

43. Dündar, E.: On rough /2-convergence of double sequences. Numer. Funct. Anal. Optim. 37(4), 480-491 (2016)

44. Malik, P., Ghosh, A.: Rough /-statistical convergence of double sequences. (2017). arXiv:1703.03173v2

45. Savaș, E., Patterson, R.F.: Lacunary statistical convergence of double sequences. Math. Commun. 10, 55-61 (2005)

46. Savaş, E.: On generalized double statistical convergence via ideals. In: The Fifth Saudi Science Conference (2012)

\section{Submit your manuscript to a SpringerOpen ${ }^{\circ}$ journal and benefit from:}

- Convenient online submission

- Rigorous peer review

- Open access: articles freely available online

- High visibility within the field

- Retaining the copyright to your article

Submit your next manuscript at $>$ springeropen.com 